\title{
EVIDÊNCIAS DA INTERRELAÇÃO TRABALHO/OCUPAÇÃO E HIPERTENSÃO ARTERIAL SISTÊMICA: UMA REVISÃO INTEGRATIVA
}

\author{
Evidence for the work/occupation and systemic hypertension interrelationship: an integrative \\ review
}

\section{Evidencias de la interrelación del trabajo/ocupación y la hipertensión arterial sistémica: una revisión integrativa}

\author{
Elizana de Fátima Garcia Soares \\ Universidade Federal de Mato Grosso - UFMT - Cuiabá (MT) - Brasil
}

\section{Lidiane da Silva Pardo}

Secretaria Municipal de Saúde - SMS - Cuiabá (MT) - Brasil

\author{
Alane Andréa Souza Costa \\ Universidade Federal de Mato Grosso - UFMT - Cuiabá (MT) - Brasil
}

\section{RESUMO}

Objetivo: Identificar evidências na literatura da interrelação entre trabalho/ocupação e o aparecimento da hipertensão arterial sistêmica. Métodos: Trata-se de uma revisão integrativa. As bases de dados utilizadas foram: Literatura Latino-Americana e do Caribe em Ciências da Saúde (LILACS) e Scientific Electronic Library Online (SciELO). Foram encontrados 11 estudos que contemplavam os critérios da pesquisa. Realizou-se a análise e síntese dos dados de forma descritiva e, em seguida, descreveu-se o valor das evidências adotadas para a elaboração da revisão integrativa. Resultados: Após revisão integrativa dos artigos, o valor III foi observado em quase a totalidade dos estudos, o que demonstra evidências intermediárias da relação do trabalho com o desenvolvimento da hipertensão. Conclusão: Embora abordado por poucos estudos, identificou-se os efeitos deletérios do trabalho/ocupação no aparecimento da hipertensão arterial sistêmica.

Descritores: Hipertensão; Saúde do Trabalhador; Condições de Trabalho.

\section{ABSTRACT}

Objective: To identify in the literature evidence for the interrelationship between work/occupation and the onset of arterial hypertension. Methods: This is an integrative review. The databases used were: Latin American and Caribbean Health Sciences (LILACS) and Scientific Electronic Library Online (SciELO). There were 11 studies that met the search criteria. The analysis and synthesis of data were conducted in a descriptive way, and then the value of evidence adopted for elaboration of the integrative review was described. Results: After an integrative review of articles, value III was observed in almost all studies, which demonstrates intermediate evidence for the relation between work and the development of hypertension. Conclusion: Despite being addressed by a few studies, the deleterious effects of the work/occupation on the onset of systemic arterial hypertension were identified.

Descriptors: Hypertension; Occupational Health; Working Conditions.

\section{RESUMEN}

Objetivo: Identificar en la literatura las evidencias que existen sobre la relación entre el trabajo y/o ocupación y la Hipertensión Arterial Sistémica. Métodos: Se trata de una revisión integrativa. Las bases de datos utilizadas fueron: Literatura Latino Americana y del Caribe en Ciencias de la Salud - LILACS y Scientific Electronic Library Online - SciELO. Se encontraron 11 estudios que cumplían con los criterios de la investigación. Se realizó un análisis y síntesis de los datos de modo descriptivo y en seguida se describió el valor de las evidencias adoptadas para la elaboración de la revisión integrativa. Resultados: El valor III fue observado en casi la totalidad de los estudios después de la revisión integrativa lo que demuestra evidencias intermedias de la relación del trabajo y el desarrollo de la hipertensión. Conclusión: Aunque pocos estudios hayan sido incluidos se pudo identificar los efectos deletéreos del trabajo y/o ocupación para la aparición de la Hipertensión Arterial Sistémica.

Descriptores: Hipertensión; Salud Laboral; Condiciones de Trabajo. 


\section{INTRODUÇÃO}

A hipertensão arterial sistêmica (HAS) é uma condição clínica multifatorial caracterizada por níveis elevados e sustentados de pressão arterial. Associa-se frequentemente às alterações funcionais e/ou estruturais dos órgãos-alvo e às alterações metabólicas, com consequente aumento de risco de complicações fatais ou não ${ }^{(1,2)}$. Também conhecida como pressão alta, é uma doença crônica controlável e um importante fator de risco para o desenvolvimento de doenças cardiovasculares ${ }^{(3)}$.

Cerca de 11 milhões de pessoas na América Latina e Caribe morrerão de doenças cardiovasculares, segundo estimativa realizada, até o ano de 2020, e a mortalidade por essa causa está cada vez mais prematura ${ }^{(4)}$. Estima-se que a hipertensão acomete de 5 a $10 \%$ da população com até 18 anos, ou seja, 7 milhões de crianças e adolescentes. Na população adulta a estimativa é em torno de $30 \%$, chegando a mais de $50 \%$ na terceira idade $^{(5)}$.

A HAS apresenta como principais fatores de risco o sedentarismo, o estresse, maus hábitos alimentares, excesso de peso, tabagismo e a idade ${ }^{(1,3)}$. Esses fatores, na maioria comportamentais, estão associados diretamente às doenças do coração e à hipertensão arterial.

Embora a HAS seja uma patologia com maior prevalência na população da terceira idade, observa-se, segundo a Sociedade Brasileira de Hipertensão (SBH), que, no Brasil, 70\% da população economicamente ativa é hipertensa ${ }^{(1)}$.

Sabe-se que a HAS é a patologia de maior risco para doenças cardiovasculares, que são responsáveis por elevados custos médicos e socioeconômicos para a saúde, por perdas relevantes na qualidade de vida e na produtividade ${ }^{(1)}$, além de serem responsáveis por morte prematura ou sobrecarga na demanda dos serviços assistenciais ${ }^{(6)}$, podendo causar ausência prolongada do empregado, comprometimento da concentração e desempenho no trabalho devido aos seus sintomas acompanhantes ${ }^{(7)}$.

É fundamental avaliar os fatores de risco cardiovascular entre a população brasileira para servir de subsídio na construção de políticas e ações de eliminação dos fatores passíveis de controle daqueles que não podem ser eliminados.

O objetivo deste estudo foi identificar evidências na literatura da interrelação entre trabalho/ocupação e o aparecimento da hipertensão arterial sistêmica.

\section{MÉTODOS}

Trata-se de uma revisão integrativa com a seguinte questão norteadora: Qual a evidência dos fatos científicos sobre o efeito do trabalho no aparecimento da hipertensão arterial sistêmica?

A revisão integrativa é definida como um método de avaliação específico que resume literatura empírica ou teórica com estudos de diferentes abordagens ${ }^{(8)}$ e tem a finalidade de fornecer maior compreensão de um determinado fenômeno ou problema de saúde, além de apresentar entendimento mais abrangente para as práticas e políticas de saúde ${ }^{(9)}$.

A busca foi através de literatura disponível em bases de dados na internet, por meio da Biblioteca Virtual em Saúde (Bireme). As bases utilizadas para a pesquisa foram a Literatura Latino-Americana e do Caribe em Ciências da Saúde (LILACS) e Scientific Electronic Library Online (SciELO), no mês de julho de 2015.

Para a busca de dados, utilizaram-se como descritores: hipertensão, saúde do trabalhador e condições de trabalho, e suas respectivas traduções para os idiomas inglês e espanhol; e realizou-se o cruzamento entre eles. Os critérios de inclusão definidos para o estudo foram: artigos voltados para a questão norteadora; ser publicado entre o período de 2009 e 2015; em um dos idiomas (português, inglês e espanhol) e estar disponibilizado na íntegra.

Prosseguiu-se com a leitura dos títulos e resumos para identificar se contemplavam e retratavam os fatores relacionados à temática. Verificou-se a disponibilidade do texto na íntegra. A busca e a seleção foram realizadas em dois momentos diferentes para que se pudesse ter maior fidedignidade na busca e inclusão dos artigos para o estudo.

A amostra total foi de 172 estudos, dos quais apenas 38 foram selecionados para compor a amostra da revisão, sendo que 24 destes foram excluídos, pois fugiram da temática, o texto não se encontrava disponível ou eram teses. Além disso, três se repetiram nas bases de dados. A análise e síntese dos dados foram realizadas de forma descritiva, levantando as informações relevantes sobre o conhecimento produzido e o tema explorado na revisão.

A seguir, descreveu-se o valor das evidências adotadas para a elaboração da revisão integrativa do presente estudo: no valor I, as evidências são provenientes de revisão sistemática de múltiplos experimentos controlados randomizados, bem delineados; no valor II, as evidências são provenientes de, pelo menos, um experimento clínico randomizado delineado; no valor III, as evidências são de experimentos bem delineados, tais como estudos não-randomizados, estudos de coorte, séries temporais e estudos de caso-controle combinados; as evidências de nível IV referem-se aos estudos não-experimentais bem delineados; e, no valor $\mathrm{V}$, as evidências são provenientes de opiniões de autoridades, baseadas em evidência clínica, ou relatos de comitês de especialistas $^{(10)}$. 


\section{RESULTADOS}

Analisou-se 11 artigos, dos quais um estava no idioma inglês, três em espanhol e sete em português. Identificou-se que o tipo de estudo predominante foi o transversal. Quanto ao ano de publicação, foram bastante homogêneos, com exceção de publicação para os anos de 2014 e 2015.

Observou-se, em relação às evidências obtidas nos artigos, o valor III em quase a totalidade dos estudos, ou seja, apresentaram evidências intermediárias do trabalho na hipertensão arterial. As evidências contribuem para uma avaliação crítica dos resultados da pesquisa, além de contribuírem para a prática baseada em evidências.

Em estudo sobre a prevalência da síndrome metabólica em funcionários de uma grande rede bancária em Vitória (ES), os autores correlacionaram ocupação e desenvolvimento da síndrome metabólica ${ }^{(11)}$. Em dois estudos realizados com professores fica evidente a situação de trabalho com a presença de hipertensão arterial ${ }^{(12,13)}$. Já em estudo sobre a prevalência da doença isquêmica do coração (DIC) em adultos e fatores associados, encontrou-se que a DIC independe da condição de trabalho ${ }^{(14)}$.

A associação independente entre o trabalho noturno e o alto risco cardiovascular foi objeto de um estudo que comprovou que é maior a frequência de alto risco cardiovascular e a prevalência da hipertensão arterial em trabalhadores noturnos ${ }^{(15)}$. Profissionais de enfermagem encontram-se em situações inadequadas de trabalho e estão expostos a maiores riscos ocupacionais, segundo alguns dos resultados de estudo com equipe de enfermagem portadora de hipertensão arterial ${ }^{(16)}$.

Não se encontrou relação significativa entre a perda auditiva induzida por ruído ocupacional, sua intensidade e prolongado tempo de trabalho com a prevalência de hipertensão arterial em trabalhadores de uma empresa petrolífera venezuelana ${ }^{(17)}$. Trabalhadores de alimentação coletiva são foco de estudo que descreveu, dentre outros, o perfil de saúde, sendo relatados casos de hipertensão $\operatorname{arterial}^{(18)}$.

As condições de saúde e de trabalho de motoristas de veículos de grande porte são retratadas em dois estudos. Em um deles considera-se que, em virtude do tipo de exposição, muitos motoristas tornam-se mais propensos a desenvolverem doenças cardiovasculares, incluindo a hipertensão arterial ${ }^{(19)}$. No outro estudo, por sua vez, comprovou-se a associação entre o nível de estresse e o surgimento de hipertensão $\operatorname{arterial}^{(20)}$.

Tendo como sujeitos de estudo somente mulheres, um estudo associou estresse no trabalho com hipertensão arterial. Os resultados mostraram risco maior de desenvolver hipertensão arterial em mulheres que realizam trabalhos passivos ${ }^{(21)}$.

Os resultados da síntese dos artigos selecionados são apresentados no quadro I a seguir. 


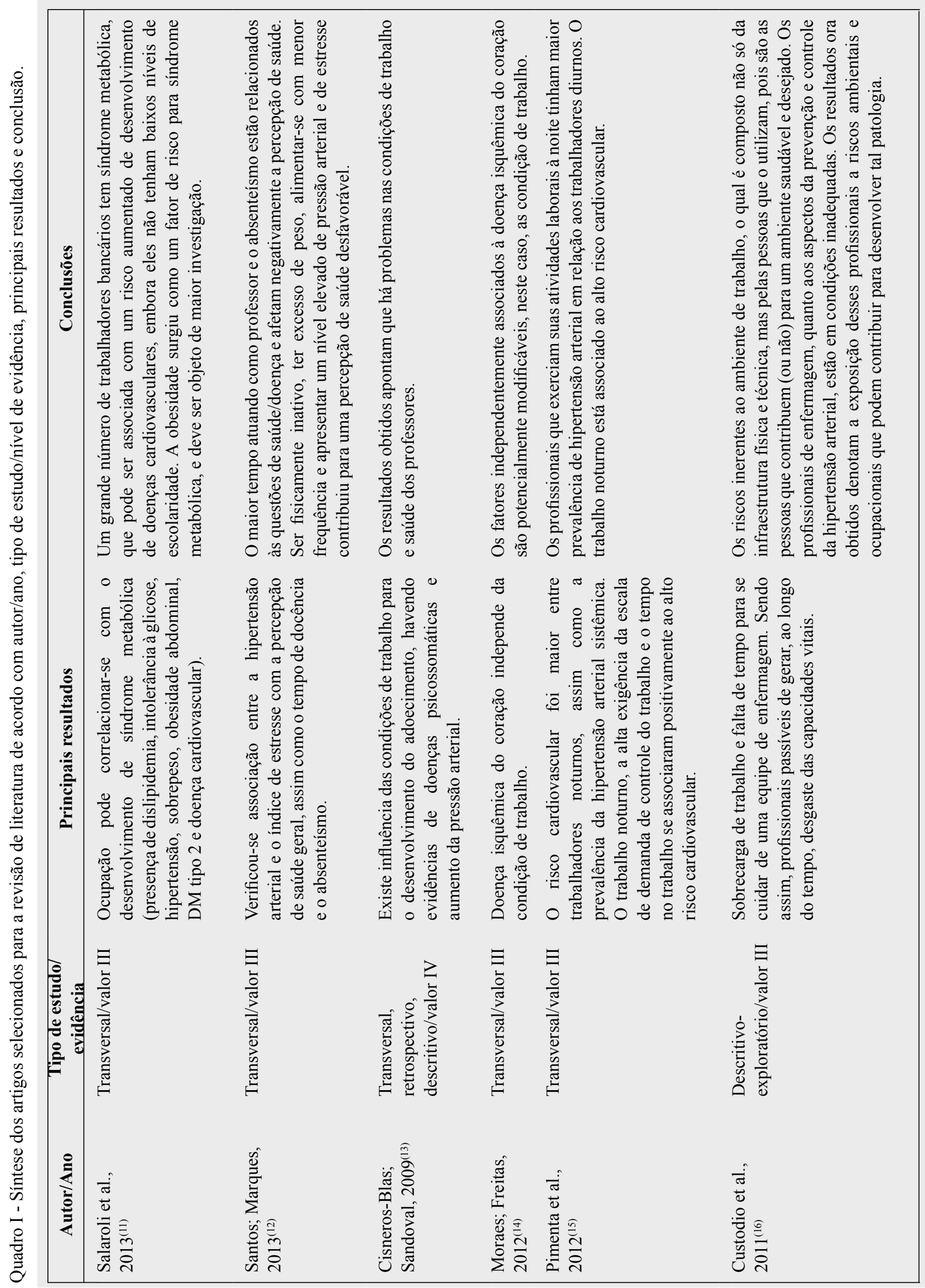




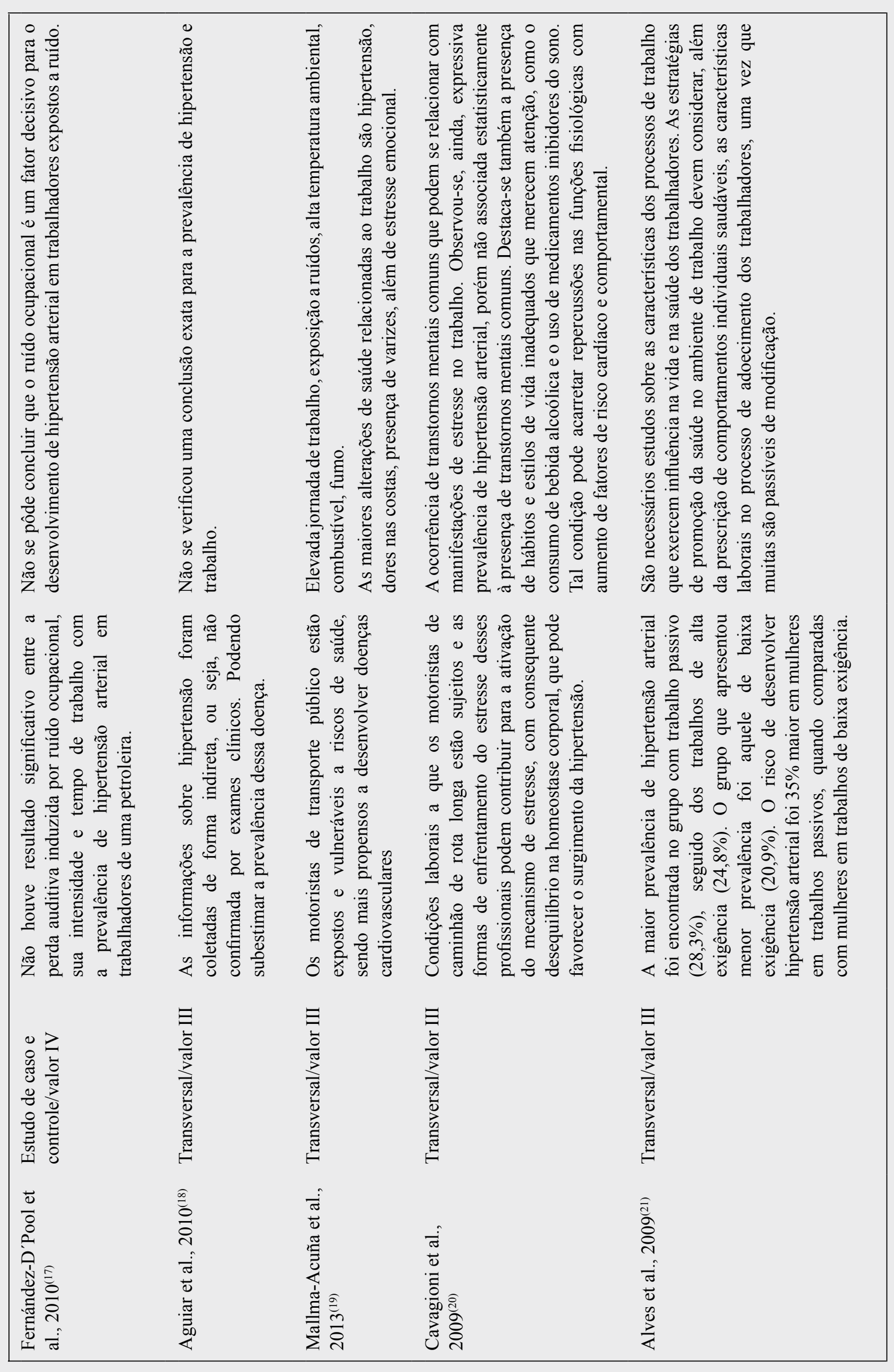




\section{DISCUSSÃO}

A hipertensão é uma patologia de elevada prevalência na população adulta ${ }^{(22,23)}$, isto é, na idade produtiva, o que implica menor produtividade no trabalho ${ }^{(24,25)}$.

As concessões de benefícios previdenciários por doenças cardiovasculares vêm aumentando. Uma das causas é a alta prevalência da HAS, condição clínica que, embora seja de diagnóstico relativamente fácil e é passível de prevenção e controle, possui expressiva ocorrência de aposentadoria por incapacidade, fato preocupante para o país ${ }^{(25)}$.

A interrelação entre o trabalho ou exposição ocupacional e a hipertensão/doenças cardiovasculares tem sido observada antes mesmo da década de 1980, porém, ainda é difícil compreender a sua etiologia quando seu surgimento envolve o trabalho ${ }^{(26)}$.

O Ministério da Saúde tem a condição do trabalho como possível causa de algumas doenças do sistema circulatório, por exemplo, hipertensão arterial, infarto agudo do miocárdio, arritmias, dentre outras. Problemas como estresse decorrente do emprego e/ou desemprego é considerado fator de risco para o desenvolvimento de $\mathrm{HAS}^{(27)}$.

Comprovou-se, em um estudo, associação positiva na exposição a níveis elevados de ruídos, pois esse trabalhador pode desencadear respostas cardiovasculares, como aumento da pressão sanguínea, provavelmente mediado pelo aumento da resistência vascular periférica ${ }^{(28)}$. Em um outro estudo mais recente observou-se que o indivíduo exposto a níveis elevados de ruídos apresenta maiores chances de ter hipertensão arterial do que o não exposto ${ }^{(29)}$.

A exposição contínua ao estresse psicossocial, a duração da jornada de trabalho e o trabalho noturno contribuem direta ou indiretamente para o aparecimento e agravamento das doenças cardiovasculares, além de levar à redução precoce da vida produtiva dos indivíduos ${ }^{(24)}$. As condições de trabalho de motoristas de caminhão de rota longa também esteve associado à doença crônica, objeto do estudo ${ }^{(20)}$.

Outro fato relevante é o trabalho noturno, que aumenta potencialmente a ocorrência das doenças cardiovasculares, o que está correlacionado ao tabagismo e à hipertensão, sendo que esta última ocorre com maior frequência nesses trabalhadores quando comparados aos trabalhadores diurnos ${ }^{(15)}$. Em estudo longitudinal realizado com japoneses que exerciam atividades laborais noturnas verificou-se aumento significativo da pressão sistólica e diastólica ${ }^{(30)}$.

Em Pernambuco, estudo com hipertensos assistidos pela Estratégia de Saúde da Família observou associação positiva do controle da HAS com as variáveis sexo, idade, nível de estudo, índice de massa corporal e ocupação ${ }^{(31,32)}$. A ocupação em rede bancária também se correlaciona ao risco de desenvolver doenças cardiovasculares, incluindo a $\mathrm{HAS}^{(33)}$.

Uma análise de forma ampliada aponta que os fatores considerados modificáveis, como excesso de peso, obesidade, inatividade física (sedentarismo), má alimentação, tabagismo, ambiente de trabalho, estresse, ocupação, dentre outros, aparecem nos estudos como fatores que contribuem significativamente para o desenvolvimento e complicação de $\operatorname{HAS}^{(17,32)}$.

Estudos mencionam a necessidade de reforçar ações de promoção e prevenção a fim de intervir no curso da HAS e controlar o impacto socioeconômico causado pelo seu agravamento ${ }^{(29)}$.

\section{CONCLUSÃo}

Poucos são os estudos que abordam a relação entre trabalho/ ocupação e o desenvolvimento da Hipertensão Arterial Sistêmica, porém foi possível identificar implicações negativas do trabalho/ocupação no aparecimento dessa patologia, embora a análise de evidências possa ser inviabilizada devido à coleta dos dados ter sido realizada, em sua maioria, em apenas um momento. Isto posto, mais pesquisas sobre a temática são necessárias a fim de obter mais informações sobre o processo saúde/ doença dos trabalhadores. Assim, poderão ser implantados programas e ações mais efetivos no ambiente de trabalho, voltados para a qualidade de vida e saúde dos trabalhadores.

\section{REFERÊNCIAS}

1. Sociedade Brasileira de Hipertensão. VI Diretrizes Brasileiras de Hipertensão Arterial. Rev Hipertensão. 2010;13(1):2746.

2. Williams B. The year in hypertension. J Am Coll Cardiol. 2009;55(1):65-73

3. Ministério da Saúde (BR), Ministério do Planejamento, Orçamento e Gestão Instituto Brasileiro de Geografia e Estatística - IBGE, Diretoria de Pesquisas Coordenação de Trabalho e Rendimento. PND - Pesquisa Nacional de Saúde 2013: percepção do estado de saúde, estilos de vida e doenças crônicas no Brasil, Grandes Regiões e Unidades da Federação. Rio de Janeiro: IBGE; 2014.

4. Ordúñez P, Silva LC, Rodríguez MP, Robles S. Prevalence estimates for hypertension in Latin America and the Caribbean: are they useful for surveillance? Rev Panam Salud Pública. 2001;10(4):226-31. 
5. Sociedade Brasileira de Hipertensão - SBH. Notícias. Sedentarismo afeta quase a metade da população brasileira, alerta Sociedade Brasileira de Hipertensão [acesso em 2015 Ago 27]. Disponível em: http://http://www.sbh.org.br/geral/noticias. asp?id $=476$

6. Silva CRS, Vieira MA, Sena RR de. Significados e reflexões sobre o ser portador de hipertensão arterial. Rev Min Enferm. 2008;12(3):346-55.

7. Nwankwo T, Yoon SS, Burt V, Gu Q. Hypertension Among Adults in the United States: National Health and Nutrition Examination Survey, 2011-2012. NCHS Data Brief. 2013(133):1-8.

8. Broome ME. Integrative literature reviews for the development of concepts. In: Rodgers BL, Knafl KA, editors. Concept development in nursing. Philadelphia: Saunders: 1993. p. 231-50.

9. Whittemore R, Knafl K. The integrative review: updated methodology. Methodological issues in nursing research. J Adv Nurs. 2005;52(5):546-53.

10. Pereira AL, Bachion MM. Atualidades em revisão sistemática de literatura, critérios de força e grau de recomendação de evidência. Rev Gaúch Enferm. 2006;27(4):491-8.

11. Salaroli LB, Saliba RAD, Zandonade E, Molina MCB, Bissoli NS. Prevalence of metabolic syndrome and related factors in bank employees according to different defining criteria, Vitória/ES, Brazil. Clinics (São Paulo). 2013;68(1):69-74.

12. Santos MN, Marques AC. Condições de saúde, estilo de vida e características de trabalho de professores de uma cidade do sul do Brasil. Ciênc Saúde Coletiva. 2013;18(3):837-46.

13. Cisneros Blas Y, Ramirez Sandoval, MLP. Prevalencia de enfermedades en trabajadores académicos de una universidad pública según seguro de gastos médicos. Salud Trab (Maracay). 2009;17(2):121-31.

14. Moraes SA, Freitas ICM. Doença isquêmica do coração e fatores associados em adultos de Ribeirão Preto, SP. Rev Saúde Pública. 2012;46(4):591-601.

15. Pimenta AM, Kac G, Souza RRC, Ferreira LMBA, Silqueira SMF. Trabalho noturno e risco cardiovascular em funcionários de universidade pública. AMB Rev Assoc Med Bras. 2012;58(2):168-77.

16. Custodio IL, Lima FET, Almeida MI, Silva LF, Monteiro ARM. Perfil sociodemográfico e clínico de uma equipe de enfermagem portadora de Hipertensão Arterial. Rev Bras Enferm. 2011;64(1):18-24.

17. Fernández-D'Pool J, Butrón J, Colina-Chourio J. Efecto del ruido sobre la presión arterial en trabajadores de una empresa petrolera venezolana. Invest Clin. 2010;51(3):301-14.

18. Aguiar OB, Valente JG, Fonseca MJM. Descrição sócio-demográfica, laboral e de saúde dos trabalhadores do setor de serviços de alimentação dos restaurantes populares do Estado do Rio de Janeiro. Rev Nutr. 2010;23(6):969-82.

19. Mallma-Acuña A, Rivera-Yngunza K, Rodas-Simbron K, Farro-Peña G. Condiciones laborales y comportamientos en salud de los conductores de una empresa de transporte público del cono norte de Lima. Rev Enferm Herediana. 2013;6(2): $107-14$

20. Cavagioni LC, Pierin AMG, Batista KM, Bianchi ERF, Costa ALS. Agravos à saúde, hipertensão arterial e predisposição ao estresse em motoristas de caminhão. Rev Esc Enferm USP. 2009;43(2):1267-71.

21. Alves MGM, Chor D, Faerstein E, Werneck GL, Lopes CS. Estresse no trabalho e hipertensão arterial em mulheres no Estudo Pró-Saúde: Estudo Pró-Saúde (Pro-Health Study). Rev Saúde Pública. 2009;43(5):893-6.

22. Gomes APF, Prazeres TMP, Correia MA, Santana FS, Farah BQ, Ritti-Dias RM. Respostas cardiovasculares durante o exercício de força em pacientes com doença arterial periférica. J Vasc Bras. 2015;14(1):55-61.

23. Soares EFG. Prevalência de doenças crônicas não transmissíveis em Cuiabá obtidas por inquérito telefônico - VIGITEL [dissertação]. Cuiabá: Universidade Federal de Mato Grosso; 2014.

24. Leão ALM. Síndrome Metabólica em funcionários de uma universidade pública: fatores associados e concordância entre critérios de diagnóstico [dissertação]. Belo Horizonte: Universidade Federal de Minas Gerais; 2013.

25. Cezário AC. Hipertensão arterial e doenças cardiovasculares como causas de concessão de aposentadoria por invalidez no Brasil [dissertação]. Salvador: Universidade Federal da Bahia; 2008.

26. Cordeiro R, Fischer FM, Lima EC Filho, Moreira DC Filho. Ocupação e hipertensão. Rev Saúde Pública. 1993;27(5): 380-7. 
27. Ministério da Saúde (BR), Organização Pan-Americana da Saúde no Brasil. Doenças relacionadas ao trabalho: manual de procedimentos para os serviços de saúde [Internet]. Brasília: Ministério da Saúde; 2001 [acesso em 2015 Jul 07]. Disponível em: http://bvsms.saude.gov.br/bvs/publicacoes/doencas_relacionadas_trabalho1.pdf

28. Andren L, Lindstedt G, Bjorkman M, Borg KO, Hansson L. Effect of noise on blood pressure and 'stress' hormones. Clin Sci (Lond). 1982;62(2):137-41.

29. Souza TCF. Exposição a ruído e hipertensão arterial: investigação de uma relação silenciosa [dissertação]. Rio de Janeiro: Fundação Osvaldo Cruz; 2010.

30. Fujino Y, Isso H, Tamakoshi A, Inaba Y, Koizumi A, Kubo T, et al. A Prospective cohort study of shift work and risk of ischemic heart disease in japanese male workers. Am J Epidemiol. 2006;164(2):128-35.

31. Paes IMBS. Estilo de vida e o controle da hipertensão arterial em indivíduos assistidos pela Estratégia de Saúde da Família, em Pernambuco [dissertação]. Recife: Fundação Osvaldo Cruz; 2012.

32. Barel M, Louzada JCA, Monteiro HL, Amaral SL. Associação dos fatores de risco para doenças cardiovasculares e qualidade de vida entre servidores da saúde. Rev Bras Educ Fís Esp. 2010;24(2):293-303.

33. Salaroli LB. Síndrome metabólica, resistência à insulina e fatores associados: um estudo em funcionários de uma rede bancária da grande Vitória/ES, Brasil [tese]. Vitória: Universidade Federal do Espírito Santo; 2011.

\section{Endereço para correspondência:}

Elizana de Fátima Garcia Soares

Rua Cinco, 12

Setor Centro Sul

Bairro: Morada do Ouro

CEP: 78053-218 - Cuiabá - MT - Brasil

E-mail: elizanasoares@yahoo.com.br 